\title{
An experimental framework for investigating hashgraph algorithm transaction speed
}

\author{
John James \\ john.james@westpoint.edu \\ United States Military Academy \\ Aaron St. Leger \\ aaron.stleger@westpoint.edu \\ United States Military Academy
}

\author{
Daniel Hawthorne \\ daniel.hawthorne@westpoint.edu \\ United States Military Academy \\ Joseph Sagisi \\ Joeseph.Sagisi@westpoint.edu \\ United States Military Academy
}

\author{
Katherine Duncan \\ katherine.duncan@westpoint.edu \\ United States Military Academy \\ Michael Collins \\ mdcolli@tycho.ncsc.mil \\ Department of Defense
}

\begin{abstract}
Power grids around the world have experienced a growing number of malicious cyber attacks. This paper provides an overview of recent use of the Hyperledger Fabric distributed operating system to prototype use of a permissioned blockchain consensus algorithm to trust shared state estimation and control data and another effort to alter local sensor data to destroy the integrity of the shared data. The paper also provides justification for an experiment to prototype use of Babble, a peer-to-peer network plugin using the hashgraph consensus algorithm, to share the state estimation and control data through transactions recorded in a hashgraph. A key claim of the hashgraph documentation, which is unsubstantiated without a proper academic analysis, is that the algorithm is asynchronous Byzantine fault tolerance (ABFT). Also, while the Hyperledger Fabric implementation supports thousands of transactions per second, the hashgraph algorithm documentation claims orders of magnitude more. Our experiment seeks to measure the hashgraph transaction speed and determine its suitability for improving the resilience of wide area control of the smart grid. The previous resilience research of the Anomaly Detection of Cyber Physical Systems (ADCPS) team includes research into inadvertent cyber and physical failures as well as malicious attacks. We conclude with some speculations concerning the potential impact of fast, fair, and secure sharing of data across a network of blockchains potentially interfaced using hashgraph distributed ledger technology (DLT).
\end{abstract}

\section{CCS CONCEPTS}

- Computer systems organization $\rightarrow$ Sensors and actuators; Real-time system architecture; Sensor networks; • Applied computing $\rightarrow$ Electronics.

\section{KEYWORDS}

Blockchain, Hyperledger fabric, hashgraph, distributed ledger technology (DLT), peer-to-peer, client-server, Internet of blockchains,

Permission to make digital or hard copies of all or part of this work for personal or classroom use is granted without fee provided that copies are not made or distributed for profit or commercial advantage and that copies bear this notice and the full citation on the first page. Copyrights for components of this work owned by others than ACM must be honored. Abstracting with credit is permitted. To copy otherwise, or republish, to post on servers or to redistribute to lists, requires prior specific permission and/or a fee. Request permissions from permissions@acm.org.

BlockSys'19, November 10, 2019, New York, NY, USA

(C) 2019 Association for Computing Machinery.

ACM ISBN 978-1-4503-7012-7/19/11 ..\$15.00

https://doi.org/10.1145/3362744.3363342 distributed control, wide-area control, asynchronous Byzantine fault tolerant (ABFT)

ACM Reference Format:

John James, Daniel Hawthorne, Katherine Duncan, Aaron St. Leger, Joseph Sagisi, and Michael Collins. 2019. An experimental framework for investigating hashgraph algorithm transaction speed. In 2nd Workshop on Blockchainenabled Networked Sensor (BlockSys'19), November 10, 2019, New York, NY, USA. ACM, New York, NY, USA, 7 pages. https://doi.org/10.1145/3362744. 3363342

\section{INTRODUCTION}

Engineers and scientists in many domains have been studying, designing, testing, implementing, maintaining and replacing productline architectures of computer-controlled/mixed-signal/hybrid systems for decades [26].

The evolving smart grid is comprised of interacting cyber and physical components and individual and institutional domains whose dynamic properties span a wide range of temporal, spatial, and spectral values. There has recently been much work defining smart grid frameworks to enable research, development and implementation of smart grid components [14] [11] [3]. The NIST architecture envisions interactions among at least seven smart grid domains (Generation, Transmission, Distribution, Customer, Markets, Operations, and Service Providers) [14].

Furthermore, the IEEE vision for smart grid controls recognizes that electric power utilities typically own and operate at least part of their own telecommunications systems (page 20 of [3]) and the IEEE vision for smart grid communications recognizes the inter dependencies among communication network components and control network components ( page 14 of [11] ).

Analysis of these frameworks highlight the multitude of cyber and physical components which are merging to enable more sophisticated processes and applications in power grids. "A critical component for modeling smart grids is modeling the interdependencies and interactions between the cyber and physical components" [31].

To develop robust and reliable controllers for smart grid applications it is imperative to have a controller design methodology which can accurately account for these dependencies. "A cyber-physical system (CPS) consists of computing devices communicating with one another and interacting with the physical world via sensors and actuators in a feedback loop" [2]. Earlier work of each of the ADCPS team members has been to apply a variety of modeling and simulation systems to investigate approaches for improving the 
resilience of the smart grid to cyber and physical failures, including applying CPS modeling methodology for Wide Area Control Systems (WACS).

The Anomaly Detection of Cyber Physical Systems (ADCPS) team of six institutions in five states (Figure 1) has conducted a series of experiments among the set of six institutional microgrids. The research efforts were supported for two years by the Office of Naval Research (ONR). Subsequently, joint research efforts have continued to develop scenarios of cyber and physical events (failure modes) and investigate approaches for building benchmark models and scenarios which will enable measurement of relative resilience of alternative control architectures for responding to detected cyber and physical anomalies.

Sensing and sharing of state estimation and control data is fundamental to the success of team efforts. The next section will provide the background of past efforts to apply distributed ledger technology (DLT) to improve trust in state estimation and control data to be shared among the cooperating set of institutional microgrids to achieve more resilient wide area control (WAC).

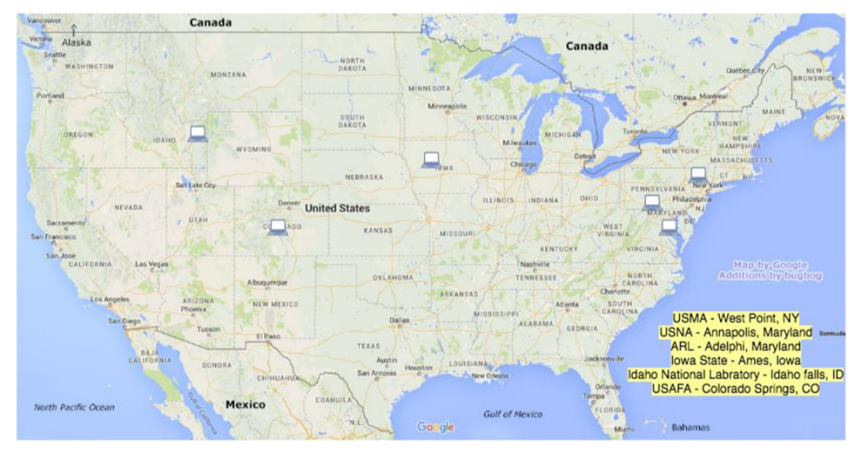

Figure 1: ADCPS team microgrid emulators/simulators.

\section{BACKGROUND}

The interdependencies among the national infrastructure of communication grids and the national infrastructure of power grids has been recognized for some time. A primary reason for the ADCPS team members to come together was to participate in a joint effort to conduct experiments in detecting and reacting to events within local microgrids which affects events occuring among the set of microgrids (i.e. cooperative data sharing which affects the distributed state estimation and control problem). The ADCPS team members all have ongoing efforts at using machine learning technologies to detect and react to cyber and physical anomalies (failure modes). The ADCPS team has also had several discussions about the need to achieve non-repudiation of data shared among team members for use in detecting and reacting to a wide variety of malicious attacks, including insider attacks and man-in-the-middle attacks.

\subsection{ADCPS Institutional Efforts}

2.1.1 Resilient power grid efforts. Dr. Craig Rieger of Idaho National Laboratory has led a series of resilience Week annual meetings to share INL resilient control results and enable other laboratories to share their resilient control results [34]. The first face-to-face meeting of the ADCPS team to discuss joint research interests in creating a benchmark model for measuring relative resilience of alternative control architectures to cyber and physical anomalies was held in conjunction with Resilience Week 2017.

2.1.2 Data sharing efforts. Trustworthy flowing of state estimation and control data across security barriers of cooperating institutions is fundamental to achieving resilience of wide area control implementations to local and global cyber and physical anomalies. Initial team efforts between the Army Research Laboratory (ARL) and Iowa State University (ISU) linked simulations running at these two institutions. An effort between USMA and other institutions is described in section 2.3 below. Dr. Glen Dudevoir of the United States Air Force Academy (USAFA) has made his test bed available for remote monitoring. Dr. Daniel Opila of the United States Naval Academy (USNA) hosted a meeting which focused on anomaly detection and analysis of shared data. Dr. Edward Shaffer of ARL has kindly offered to make data from ADCPS team experiments in wide area control available through an ARL data repository for other laboratories to access and conduct their own experiments to verify the results.

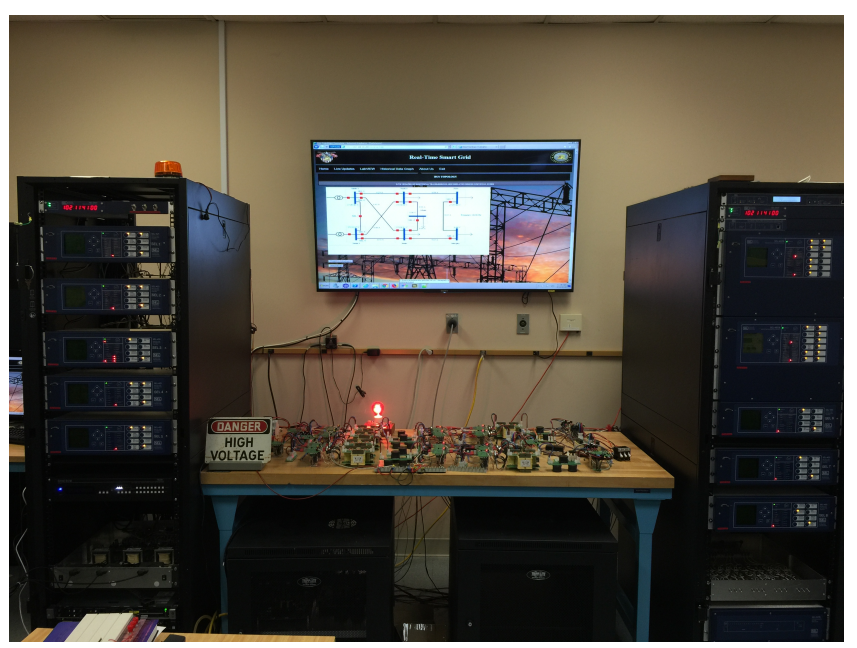

Figure 2: Smart Grid Test Bed

\subsection{USMA Smart Grid Emulator}

For the last several years students and faculty at USMA have been using a microgrid emulator (Figure 2) to conduct experiments in Wide Area Monitoring and Control (WAMC) [32]. The testbed has seven Phasor Measurement Units (PMUs) which collect data . All the PMU data is consolidated in an SQL database by OpenPDC.

Two recent faculty and student efforts are described below which used the testbed and coordinated the projects with the ADCPS.

\subsection{USMA Hyperledger Fabric DLT prototype}

One effort was to ensure that data shared among the ADCPS team members regarding each institution's state estimation and control data was non-refutable as being received from each of the team members. Hyperledger Fabric distributed ledger technology (DLT) 
was used [10] to apply a consensus protocol which resulted in each participating node receiving a copy of each transaction. Thus, each node maintained a current copy of a synchronized estimate of the global system state stored in OpenPDC [35] [1] data bases located at each participating node. Last year a student and faculty team developed a prototype of an approach for using the Hyperledger Fabric distributed operating system [17] to apply a permissioned blockchain consensus algorithm to share state estimation and control data among among the ADCPS team members. [10]

2.3.1 ADCPS power grid network. For the ADCPS set of microgrids (or for any set of peers sharing local state of a distributed control system) we need a set of local nodes sharing valued information across a larger network. In our case the larger network is a set of six microgrids selectively sharing data over the Internet.

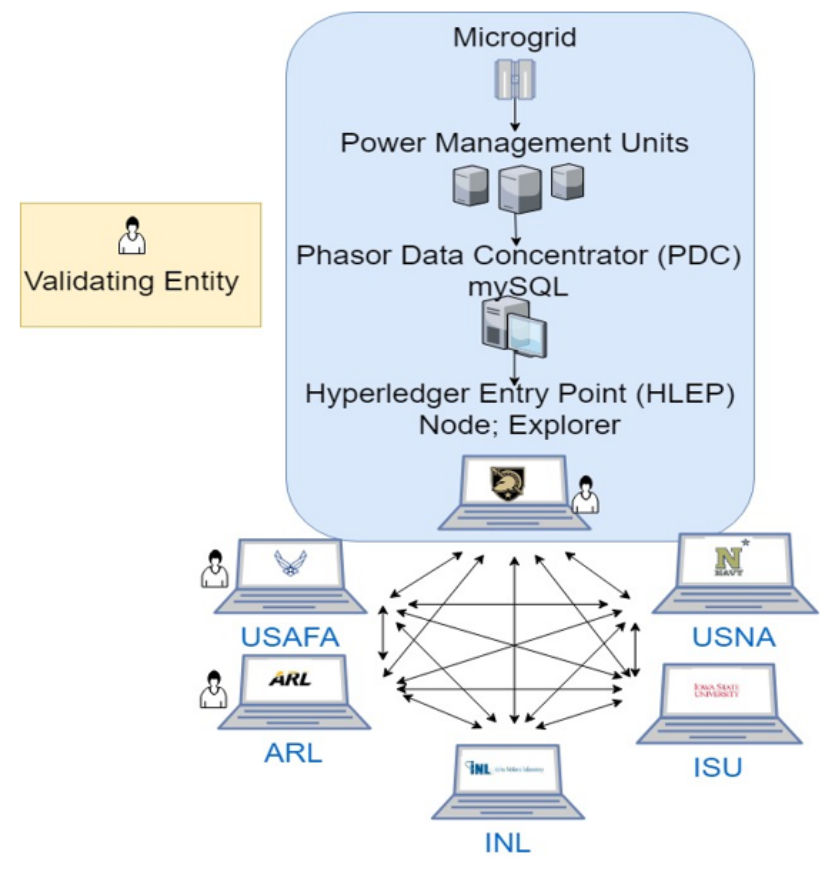

Figure 3: ADCPS wide area network of six microgrids.

2.3.2 Internal USMA network. And a more detailed version of the USMA local network components is shown in figure 4.

2.3.3 Making data available to Hyperledger Fabric for consensus. The process of data retrieval from a node in the network follows the flow of the USMA network component shown in Figure 4. MySQL data from the USMA testbed is read by one of the hosts located at USMA. The data from that PC is read by the database OPVN client (Node). Data is obtained by running a database query algorithm discussed in the next subsection. Then from the OPVN client, data will be sent out the VPN concentrator with an update request to the rest of the nodes on the network, and the nodes will confirm the request to update the ledger. The other nodes are then able to connect to the VPN concentrator in order to send confirmation requests back to our client. Once the request is sent back to our

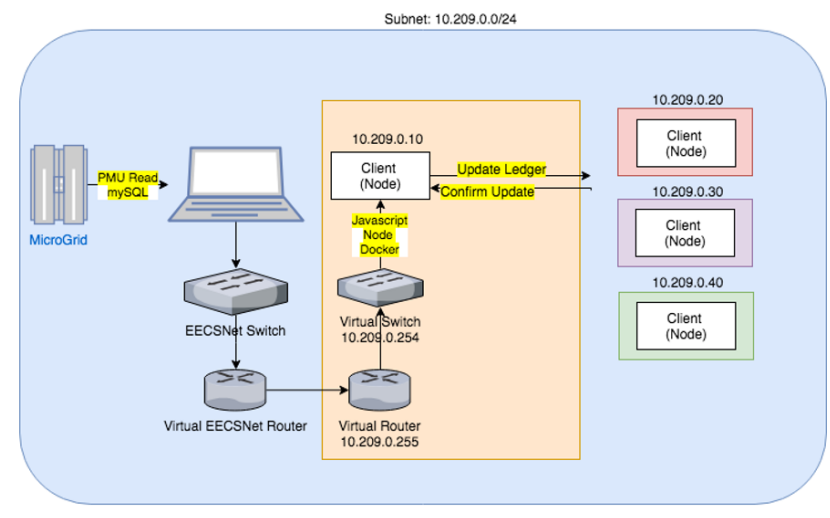

Figure 4: Details of USMA local area network components.

client node validating the update, the ledger state is updated with the appropriate information. Each node on the network will run a similar instance of OPVN client node as is done for the USMA node.

Each node provides the local state estimation and control data for a given transaction and the consensus process results in each node having a copy of the data provided by all of the nodes which participated in a given consensus transaction. The sequence of transaction entries present at each participating peer microgrid comprise the distributed ledger.

2.3.4 Database query of OpenPDC data. We used PyMySQL and JSON modules. The PyMySQL module enables us to interface with the SQL database [29]. The JSON module acts like an encoder and decoder to help us convert our data into a useable JSON format [16]. The python script first establishes a connection to the testbed SQL database using proper authentication. After successfully connecting, the script obtains information related to PMU data from a text file. The text file contains unique identifiers for all PMU measurements and applicable variables necessary for queries. The program then loops through each PMU and queries the SQL database for the latest readings. The queries are written to a file in readable format. Additionally, each query is printed to the console (for verification that the script is working). After completing the above actions, the script closes all files and connections. In order to more easily create historic data for our network, the script is being updated to accommodate JSON formatting.

2.3.5 Hyperledger Fabric Project results. The project successfully built the prototype information sharing prototype using the Hyperledger Fabric DLT consensus algorithm and successfully queried the OpenPDC database to collect state estimation and control data placed into the MySQL database from the PMU sensors. The project team also successfully set up OpenVPN communication channels over the Internet to two of the other five ADCPS team members and pinged the other microgrid local networks to collect network latency data. However, time expired before the student team could demonstrate shareing state estimation and control data with the other team members. 


\subsection{USMA Man-in-the-middle data packet alteration prototype}

While the project described above sought to avoid man-in-themiddle attacks between institutions,a second project sought to invalidate sensor data being stored within institutional nodes. That is to implement a man-in-the-middle attack on the validity of the data being reported by a Sweitzer Engineering Laboratory (SEL) synchrophasor sensor into the local OpenPDC database using an FPGA to alter data in network packets at network speed. [18] As indicated in the upper left hand corner of Figure 4, the data being shared using the (very trustworthy) Hyperledger Fabric consensus algorithm, is being acquired from the OpenPDC MySQL database after the data is collected from the set of SEL synchrophasor sensors. So a physical attack was created in a second project to alter data being reported from the sensors by altering appropriate data packet values [18].

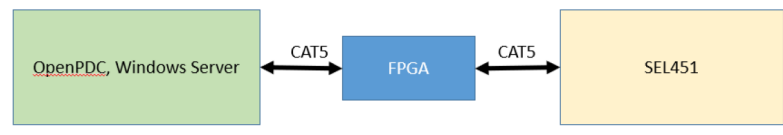

Figure 5: FPGA inserted into Testbed Network

2.4.1 Man-in-the-middle attack hardware. As of the end of the semester, we have not moved to testing our attack on the testbed's real hardware. Instead, we simulated inserting a DE2-115 Altera Board inline, between an SEL451 PMU and the Windows server running OpenPDC, as shown figure 5 above, the field programmable gate array is configured to be placed between the WIndows server running the OpenPDC data concentration service and the SEL451 sensor providing the current, voltage, timestamp and phase angle data values. Thus, all packets transmitted between the PMU and the server have to pass through, and be intercepted by, the DE2-115 board.

2.4.2 Man-in-the-middle attack results. The project successfully simulated the use of the Altera FPGA altering PMU data packets by using ModelSim 10.4. This required programming a synchrophasor packet generator in ModelSim using information from Wiresharkcaptured packets on the testbed's network. We programmed ModelSim to generate packets using the structure of packets found on the network of our testbed. This modeled an SEL451 PMU sending data frames to the PDC. ModelSim allowed us to simulate Verilog code as if it was being written to our Cyclone IV FPGA.

The project successfully developed three attacks to test exerting increasing amounts of control over packets as they passed through the processor. For the initial attack, all of the bits of the data frame that were associated with measurement data were replaced with zeroes to show that we had arbitrary control over the phasor values presented to the PDC. We called this a "constant measurement value attack." In the next attack, which we called a "variable measurement value attack," we subtracted 1 bit each from the binary values for real and imaginary voltages to prove that the phasor values could be modified based on their original value, rather than just replaced.

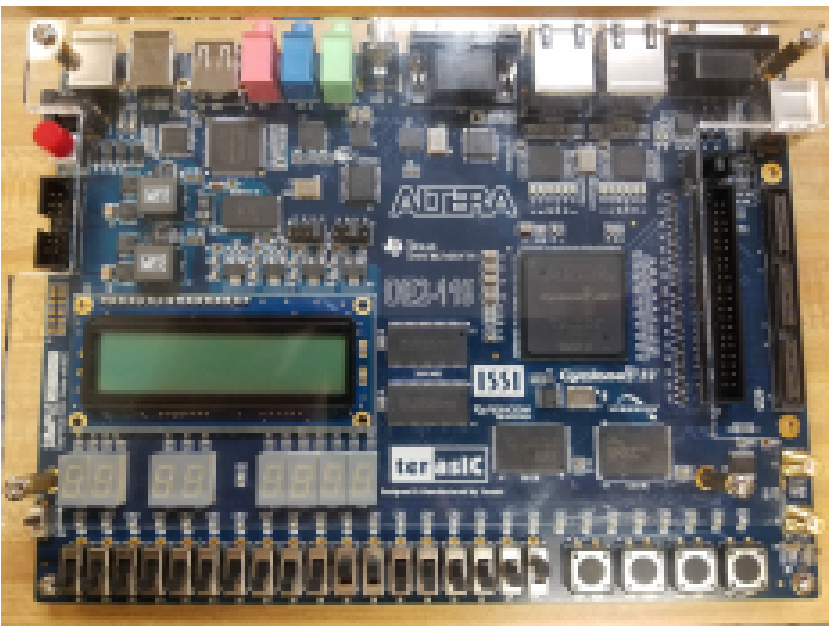

Figure 6: Altera DE2-115 Development and Education Board

Finally, we performed what we called a "time shift attack," in which we initially saved the measurement data from the first packet, then sent that packet through unchanged. Every following packet received the measurement data of the preceding packet, creating the illusion that all of the PMUs measurements were occurring one packet later than they actually were. Ideally, each of these attacks would also have included replacing the synchrophasor $\mathrm{CHK}$ bytes at the end of the packet with a newly calculated checksum, thereby making the packet appear unmolested. Unfortunately, time constraints on our research ended up forcing us to forgo this important step for the time being.

The man-in-the-middle attack project demonstrated the risks created by the lack of strong integrity and authentication checks in the IEEE Synchrophasor Protocol. The simulation of the attacks proves that it is plausible to use an FPGA to modify Synchrophasor packets, while minimizing the effect of latency, in order to fool a PDC into accepting faulty data from a PMU. If these attacks were successfully executed on a production system, an attacker could not only insert arbitrary values for each measured magnitude and phase, but could also increase or decrease these values by a certain amount to achieve a specific desired effect, create the illusion that a generator is out of phase, or even completely replay captured data in a loop.

\section{ADCPS RESEARCH INTERESTS AND HASHGRAPH DLT PROTOTYPE}

At the last meeting of the ADCPS team an agreement was reached regarding future joint research interests:

- student and faculty projects with commercial and/or government laboratory sponsors acting as the "customer" for student multidisciplinary teams,

- projects including use of microgrid emulators as vehicles for hardware-in-the-loop and software-in-the-loop experiments, and

- projects with possible overlaps with other application domains (such as the satellite communications domain) of the technical results of considerations of resilience of the smart grid domain as a 
composite problem of interdependencies of resilient communication networks interacting with resilient control networks with both being guided by social/cognitive networks.

Executing netted, distributed control experiments in these joint research areas will require an open solution to sharing state estimation and control data across institutional security boundaries. USMA is building upon the lessons learned from the Hyperledger Fabric DLT prototype effort to build a second DLT prototype to support improvements in the trust in shared visualizations of distributed system state estimation and control data.

\subsection{State of the art in use of distributed ledger technology}

3.1.1 Papers from BlockSys 2018. The papers presented at BlockSys 2018 were reviewed as part of the state of the art review conducted for the prototype effort. The papers were each individually impressive and offered insights into approaches for applying DLT for sharing data among a set of peers [12] [25] [13] [30].

3.1.2 Choice of hashgraph DLT for next prototyping effort. Wide area control of the smart grid requires cyclic updates of distributed state estimates of grid energy demanded by consumers and cyclic changes in energy provided by grid generator response which must be sufficient to ensure stability of the grid operating at $60 \mathrm{HZ}$ and providing 120 volts at wall outlets. Hyperledger Fabric was chosen for the initial DLT implementation for sharing state estimation and control data based upon the open source nature of the software and the documented transaction speed. The Hedera Hashgraph implementation has been selected for the follow on experiment based upon the documented claims of orders of magnitude faster transaction speed than Hyperledger Fabric and the decision of IBM (the initial creator of Hyperledger Fabric) to join hedera hashgraph and enable use of the hashgraph algorithm to provide a consensus transaction service for Hyperledger Fabric blockchain implementations [9]. Also, the Hedera hashgraph whitepaper makes the claim that the Hedera hashgraph algorithm is the only DLT consensus algorithm to be asynchronous Byzatine fault tolerant (ABFT) [7].

A hashgraph is a cryptographic hash of a directed acyclic graph (DAG) so is termed a "hashgraph." To build a linear sequence of blocks to form a blockchain the hashgraph events must be projected into a series of blocks. So a given hashgraph is not strictly a blockchain but a given linear sequence of blockchain blocks can be uniquely constructed from a given hashgraph.

While the ABFT claim of the hashgraph whitepaper has been substantiated by a machine COQ proof constructed from the whitepaper by Professor Karl Crary of CMU, [20] , the claim is unsubstantiated without a proper academic analysis. However, while we expect that the ABFT claim will be subjected to proper academic analysis at some time in the future, the goal of our current experiment is not to provide an analysis which can confirm the ABFT claim. The goal of our experiment is to determine if the hashgraph algorithm is capable of supporting a smart contract service which will enable selective sharing of local microgrid state estimation and control data among a permissioned set of cooperating peers fast enough to support improved resilience of a wide area control solution for the set of cooperating microgrids. Also, use of a smart contract process to enable resilient wide area control of the smart grid is not known to be subject to formal proofs since a proof process has not been developed for distributed cyber physical systems (CPS). That is, formal methods alone are incapable of proving the occurrence (much less the resilience) of the observability, controllability, stability and safety properties of CPS [4], [19], [23], [24]. While we anticipate the DLT service to be extremely valuable in verifying the trust and provenance properties of shared state estimates, the trust property alone is insufficient to verify the required control system properties which need to be verified to achieve resilience. These properties span several orders of magnitude of temporal, spatial, and spectral scale constraints across the different smart grid control hierarchies and interdependent domains.

Our hashgraph DLT prototype will use a mesh network ( perhaps several Pycom devices [28] and/or several Raspberry Pi devices [27]) to establish an open source ad hoc mesh network service at the transport layer and use an open source hashgraph algorithm implementation [22], [5] as a middleware layer between the mesh network and the data transport service application layer. The initial design is a local mesh network with five or six nodes in a single room with each node sharing data from a single temperature sensor.

Our experiment seeks to measure the capability of the hashgraph DLT algorithm to rapidly achieve shared situational awareness among a set of peer nodes of room temperature based upon a voting scheme for reported temperatures. However, we expect that if we use a heat lamp to cause one or more of the temperature sensors to report bad data, then we should immediately be able to flag the compromised sensor(s) and experimentally verify the ability of the framework to provide out-of-band identification of faulty sensors.

Also, sensor data can be used to create sensor profiles for maintenance purposes and time stamps can be used to prevent manin-the-middle attacks based upon lateness of data reported from compromised nodes while voting schemes can be used to identify man-in-the-middle attacks such as the one described in section 2.4 above.

In addition, the hashgraph documentation claims the potential of applying Inter-Blockchain Communication Protocols to enable data sharing among public permissioned ledgers, public permissionless ledgers, private permissioned ledgers, and private permissionless ledgers [8]. Thus, hashgraph interfaces are feasible among the different domains (knowledge silos) of the NIST smart grid framework (Generation, Transmission, Distribution, Customer, Markets, Operations, and Service Providers).

\subsection{USMA Hashgraph DLT prototype}

Our team believes that the current blockchain and hashgraph technologies are set to enable improving the trustworthiness of concurrent estimates of the communication networks, the control networks and the social-cognitive networks associated with resilient control of critical infrastructures.

Thus the next three sub-sections provide comments on the Swirlds platform [33], the Babble platform [5], and a summary of the status of prototype efforts to leverage the orders of magnitude increase in transaction speed of the hashgraph consensus algorithm to improve the resilience of the smart grid. While that lofty resilience goal is our ultimate aim, the initial intent of the prototype is to establish non-repudiation of temperature data being shared by a 
set of sensors streaming their data over a MANET and detect when one or more of the temperature sensors is providing bad data.

3.2.1 Swirlds platform. The Swirlds platform software development (SDK) is available to download from the Swirlds web site. Swirlds implements the hashgraph consensus algorithm developed by Leemon Baird. The Swirlds platform hashgraph algorithm has been machine proven to be ABFT compliant.

3.2.2 Babble platform. The Babble platform software development (SDK) is available to download from the Babble github site [6].

3.2.3 Prototype status. We have an initial concept for design of the MANET communication network and the sensors to be connected and have most of the components on-hand. While certainly not necessary for the initial prototype, several team members were previously involved in sharing data across security boundaries using need-to-share information security policies [24] and the intent is to use lessons learned from the prototype to share the design with other ADCPS team members and apply the design of figures 3 and 4 to share smart grid state estimation and control data while supporting increased trust in the data used for the wide-area control solution in responding to cyber and physical anomalies..

\section{CONCLUSIONS AND FUTURE WORK}

A current gap in improving resilience of large-scale distributed systems is the growing lack of trust in the data shared among cooperating peers. This trust must start with ensuring the provenance of data from the lowest level of sensors and actuators in the communications and control hierarchies. Unfortunately, current solutions for achieving and maintaining trust in the provenance of sensor and actuator values are increasingly vulnerable.

Hashgraph DLT [20] implementations, such as the Swirlds hashgraph platform or the Babble hashgraph platform, may have the potential to meet the "need for speed" of sharing local state estimation and control data among cooperating microgrids while addressing the current shortfall regarding lack of trust in shared estimates. The Hyperledger Fabric architecture is modular in nature and a project is underway to make the faster Hedera hashgraph consensus service available to Hyperledger Fabric blockchain implementations [21]. Although the ABFT claims of the hedera whitepaper are unsubstantiated without a proper academic analysis, the goal of our experiment is to measure the transaction speed for use with sharing smart grid data among a permissioned group of peer institutions and estimate the potential for meeting the needs of achieving more resilient control of the smart grid.

Also, extending our current hashgraph prototype to share local microgrid sensor data among peer ADCPS microgrids will enable "out of band" verification of power grid frequency, voltage, phase angle and time stamp data needed by each peer to resiliently and collectively achieve distributed wide-area control. Additionally, among the social/cognitive networks of people choosing and executing information security policies, explicit decisions are needed to declare need-to-know information security policies for that data (such as privacy data for individuals or proprietary data for corporations or classified data for governments) which must remain confidential which is stored on networks.
At the same time, in order to have sufficient data available at each local node of the global system estimation and control data, the cognitive/social network at each microgrid needs to declare appropriate need-to-share information security policies implemented over peer-to-peer mobile ad hoc network (MANET) architectures and other communication architectures up to the global wi-fi networks. This will enable sharing the data needed to achieve joint real-time state estimation and control goals/targets across institutional security boundaries.

The information security policy makers at each cooperating institution must explicitly separate the need-to-know security policies from the need-to-share policies to ensure that proper protections are maintained for privacy/proprietary/classified data while proper protections are also maintained to ensure that a compromise of the declared information sharing policies are not committed which prevent sharing across institutional information security boundaries that critical data needed to meet joint cooperative control goals/targets.

It then becomes feasible to set local and global information security policies among a set of peer microgrids (or a set of peer distributed nodes in any domain) to enable "out-of-band" analysis of anomalies of interest (such as sensor failure or sensor compromise or other insider failures/attacks) and collective response to detection of and reaction to such anomalies. Such efforts will be made easier if the Hedera Hashgraph vision of a fully-decentralized, permissionless, public ledger (potentially of millions of nodes) comes to pass since oracles can then be constructed which provide streams of expert data required to support such analyses [8].

For real-time, distributed systems the most important feature of the hashgraph consensus algorithm in improving the resilience of distributed control systems may well be the speed with which the consensus algorithm is able to report the time at which consensus is achieved [21]. That is, the hashgraph consensus algorithm returns the fair ordering of a set of messages comprising a transaction in a distributed ledger for which each participant has a copy of the ledger entry at their own location. The algorithm which ordered the ledger entries functions without reference to a centralized clock or to a centralized ordering service (such as that currently used by Hyperledger Fabric [15]). Moreover, the ledger entry achieved by the hashgraph consensus algorithm contains a time stamp which is obtained from analysis of the times at which the distributed nodes participated in the consensus process for a given transaction entry.

Finally, the hashgraph documentation claims the hashgraph algorithm can enable data sharing among public permissioned ledgers, public permissionless ledgers, private permissioned ledgers, and private permissionless ledgers [8]. Thus, our future work will seek to confirm that capability and enable the fast, fair, and secure sharing of data across a network of blockchains potentially interfaced using hashgraph distributed ledger technology (DLT) to improve the resilience of the smart grid.

\section{ACKNOWLEDGMENTS}

This work was partially supported by the Office of Naval Research. The views expressed herein are those of the authors and do not purport to reflect the position of the United States Military Academy, the Department of the Army, or the Department of Defense. 


\section{REFERENCES}

[1] Grid Protection Alliance. 2009. Open Source Phasor Data Concentrator. Grid Protection Alliance. Retrieved August 2, 2019 from https://github.com/ GridProtectionAlliance/openPDC

[2] R. Alur. 2015. Principles of Cyber-Physical Systems. MIT Press, Cambridge, MA.

[3] Anuradha M. Annaswamy, Massoud Amin, Christopher L. DeMarco, and Tari Samad. 2013. IEEE VISION FOR SMART GRID CONTROLS: 2030 AND BEYOND. IEEE SMART GRID RESEARCH. IEEE, IEEE 3 Park Avenue New York, NY 100165997 USA. https://ieeexplore.ieee.org/abstract/document/6690098/

[4] Jean-Pierre Aubin. 2009. Viability Theory. Birkhauser, New York, NY.

[5] Babble. 2019. Babble Consensus. babble. Retrieved August 2, 2019 from https: //www.babble.io/

[6] Babble. 2019. BFT Consensus platform for distributed applications. babble. Retrieved August 2, 2019 from https://github.com/mosaicnetworks/babble

[7] Leemon Baird. 2016. THE SWIRLDS HASHGRAPH CONSENSUS ALGORITHM: FAIR, FAST, BYZANTINE FAULT TOLERANCE. Swirlds Tech Report SWIRLDS TR-2016-01. Swirlds. https://hashgraph.org/discussion/171/whitepaper-thehashgraph-consensus-algorith

[8] Leemon Baird. 2019. Our Path to Decentralization. Hedera. Retrieved August 6 , 2019 from https://www.youtube.com/watch?v=QTNNYeSks-s

[9] Leemon Baird, Bryan Gross, and Donald Thibeau. 2019. Hedera Consensus Service. Hedera. Retrieved September 18, 2019 from https://www.hedera.com/hhconsensus-service-whitepaper.pdf

[10] Christian Banks, Samuel Kim, Michael Neposchlan, Nicholas Velez, Katherine Duncan, John James, Aaron St. Leger, and Daniel Hawthorne. 2019. Blockchain for Power Grids. In 2019 IEEE SoutheastCon (SoutheastCon 2019). IEEE, New York, NY, USA.

[11] Stephen Bush, Sanjay Goel, and Georges Simard. 2013. Smart Grid Research: Communications - IEEE Vision for Smart Grid Communications: 2030 and Beyond Roadmap. IEEE Report. IEEE, IEEE 3 Park Avenue New York, NY 10016-5997 USA. https://ieeexplore.ieee.org/abstract/document/6690098/

[12] Roberto Casado-Vara, Fernando de la Prieta, Javier Prieto, and Juan M. Corchado. 2018. Blockchain framework for IoT data quality via edge computing. In Proceedings of the 1st Workshop on Blockchain-enabled Networked Sensor Systems (BlockSys '18). ACM, ACM, Shenzhen, China, 19-24. https://doi.org/10.1145/ 3282278.3282282

[13] Atis Elsts, Efstathios Mitskas, and George Oikonomour. 2018. Distributed Ledger Technology and the Internet of Things: A Feasibility Study. In Proceedings of the 1st Workshop on Blockchain-enabled Networked Sensor Systems (BlockSys '18). ACM, ACM, Shenzhen, China, 7-12. https://doi.org/10.1145/3282278.3282280

[14] Greer et al. 2014. NIST Framework and Roadmap for Smart Grid Interoperability Standards, Release 3.0. NIST. Retrieved August 1, 2019 from https://www.nist.gov/publications/nist-framework-and-roadmap-smartgrid-interoperability-standards-release- 30

[15] Hyperledger Fabric. 2019. Welcome Hyperledger Fabric - Ordering Service FAQ Hyperledger Fabric. Retrieved August 7, 2019 from https://hyperledger-fabric readthedocs.io/en/release-1.1/ordering-service-faq.html

[16] Python Software Foundation. 2019. FSON encoder and decoder. Python Software Foundation. Retrieved August 2, 2019 from https://docs.python.org/2/library/ json.html

[17] The Linux Foundation. 2019. Hyperledger Fabric. The Linux Foundation. Retrieved August 1, 2019 from https://www.hyperledger.org/projects/fabric

[18] Jared Fritz, Joseph Sagisi, John James, Aaron St. Leger, Kyle King, and Katherine Duncan. 2019. Simulation of Man in the Middle Attack On Smart Grid Testbed. In 2019 IEEE SoutheastCon (SoutheastCon 2019). IEEE, New York, NY, USA.

[19] John Gukenheimer and Philip Holmes. 1983. Nonlinear Oscillations, Dynamical Systems, and Bifurcations of Vector Fields. Springer-Verlag, New York, NY, USA.

[20] Hedera. 2018. Coq Proof Completed By Carnegie Mellon Professor Confirms Hashgraph Consensus Algorithm Is Asynchronous Byzantine Fault Tolerant Hedera. Retrieved August 2, 2019 from https://www.hedera.com/blog/coq-proofcompleted-by-carnegie-mellon-professor-confirms-hashgraph-consensusalgorithm-is-asynchronous-byzantine-fault-tolerant

[21] Hedera. 2019. The Hedera Consensus Service. Hedera. Retrieved August 7, 2019 from https://www.hedera.com/hh-consensus-service-whitepaper.pdf

[22] Hedera. 2019. It's been their internet for too long. Make it yours. Hedera. Retrieved August 2, 2019 from https://www.hedera.com/

[23] Douglas R. Hofstadter. 1979. Gödel, Escher, Bach: An Eternal Golden Braid. Basic Books, Inc, New York, NY.

[24] John R. James, Frank Mabry, and Kevin Huggins. 2012. Seeing the Real World: Sharing Protected Data In Real Time. In Proceedings of the 45th Hawaii International Conference on System Sciences (HICCS 45). IEEE, ACM, Maui, HI, 2357-2365. https://doi.org/10.1109/HICSS.2012.518

[25] Uzair Javaid, Muhammad Naveed Aman, and Biplab Sikdar. 2018. BlockPro Blockchain based Data Provenance and Integrity for Secure IoT Environments. In Proceedings of the 1st Workshop on Blockchain-enabled Networked Sensor Systems (BlockSys '18). ACM, ACM, Shenzhen, China, 13-18. https://doi.org/10.1145/ 3282278.3282281
[26] E. A. Lee and P. Varaiya. 2011. Structure and Interpretation of Signals and Systems (2nd. ed.). LeeVaraiya.org, Berkeley, CA. https://ptolemy.berkeley.edu/books/ leevaraiya/releases/LeeVaraiya_DigitalV2_04.pdf

[27] Raspberry Pi. 2019. Raspberry Pi. Raspberry Pi. Retrieved August 2, 2019 from https://www.raspberrypi.org/

[28] pycom. 2019. pycom. pycom. Retrieved August 2, 2019 from https://pycom.io/

[29] PyMySQL. 2019. PyMySQL User Guide. PyMySQL. Retrieved August 2, 2019 from https://pymysql.readthedocs.io/en/latest/user/index.html

[30] Siamak Solat. 2018. RDV: An Alternative To Proof-of-Work And A Real Decentralized Consensus For Blockchain. In Proceedings of the 1st Workshop on Blockchain-enabled Networked Sensor Systems (BlockSys '18). ACM, ACM, Shenzhen, China, 7-12. https://doi.org/10.1145/3282278.3282283

[31] Aaron St. Leger, John James, and Dean Frederick. 2012. Smart Grid Modeling Approach for Wide Area Control Applications. In Proceedings of the 2012 IEEE Power and Energy Society General Meeting (PES '12). IEEE, IEEE, San Diego, CA, 422-431. https://ieeexplore.ieee.org/document/6345511

[32] Aaron St Leger, Jeremy Spruce, Thomas Banwell, and Michael Collins. 2016. Smart grid testbed for Wide-Area Monitoring and Control systems. In 2016 IEEE/PES Transmission and Distribution Conference and Exposition (T\&D (PES T\&D 2016). IEEE, New York, NY, USA, 1-5. https://doi.org/10.1109/TDC.2016.7519995

[33] Swirlds. 2019. pycom. Swirlds. Retrieved August 2, 2019 from https://www. swirlds.com/

[34] Resilience Week. 2019. Resilience Week. Wikipedia. Retrieved August 4, 2019 from https://events.inl.gov/Resilience-Week-2019/Events/Agenda?EventId=4

[35] Wikipedia. 2009. OpenPDC. Wikipedia. Retrieved August 2, 2019 from https: //en.wikipedia.org/wiki/OpenPDC 